\title{
La enseñanza de las ciencias por indagación y el diseño ingenieril en educación primaria
}

\author{
Silvana Pérez y Jesús A. Meneses Villagrá \\ Dpto. Didáctica Ciencias Experimentales. Universidad de Burgos
}

[Recibido el 12 de mayo de 2019, aceptado el 14 de septiembre de 2020]

Aunque existen investigaciones acerca de cómo aplicar enfoques basados en la práctica científica e ingenieril en la educación primaria y secundaria, en los libros de texto de Ciencias de la Naturaleza pocas son las actividades didácticas que utilizan todas las etapas que caracterizan a estos enfoques. En este trabajo, se presenta una propuesta didáctica de la asignatura de Ciencias de la Naturaleza basada en la metodología de indagación y en el diseño ingenieril, desde la planificación de actividades secuenciadas, pasando por la aplicación y finalmente analizando en los estudiantes su evolución en el desarrollo de actitudes y emociones hacia las ciencias y habilidades científicas. El estudio se llevó a cabo en el cuarto curso de Educación Primaria durante el tercero trimestre del año lectivo 2018-2019. Fue posible percibir una evolución con respecto a las competencias científicas, limitada por el nivel de desarrollo cognitivo de los estudiantes participantes en la investigación.

Palabras clave: Actividades de aprendizaje, actividades indagatorias, competencias científicas, actitudes hacia la ciencia.

\section{Inquiry- and engineering design-based science in primary school}

Despite considerable research in the application of practice-based teaching approaches to science and engineering in primary and secondary education, natural science textbooks have been slow to incorporate such activities into their content. This article analyses the creation and implementation of a natural science teaching and learning proposal using an inquiry- and engineering designbased methodology. The study examines how the activities were designed and transferred into a classroom context, and their impact on the pupils' cognitive and emotional development of student in terms of their attitudes towards science and acquisition of scientific skills. The activities were carried out with a group of fourth-class primary school children (aged 9-10 years) during the third term of the 2018-2019 school year. The results showed an increase in the pupils' scientific skills in accordance with their level of cognitive development.

Keywords: learning activities, inquiry-based activities, scientific skills, attitudes toward.

Para citar el artículo. Pérez S. y Meneses Villagrá, J.A. (2021). La enseñanza de las ciencias por indagación y el diseño ingenieril en educación primaria. Ápice. Revista de Educación Científica, 5 (1), 1-19. DOI: https://doi.org/10.17979/arec.2021.5.1.5807

Contacto.silperez_1972@hotmail.com,meneses@ubu.es 


\section{Introducción}

Los avances científicos y tecnológicos experimentados en las últimas décadas afectan tan profundamente a la forma como la información llega a los ciudadanos, que están interfiriendo en su manera de construir el conocimiento. Según Fraiha et al. (2018), la escuela del nuevo milenio tiene el desafío de formar ciudadanos capaces de apropiarse críticamente de la información disponible en los diversos medios de comunicación y, basándose en las teorías científicas, tomar decisiones que les permitan vivir mejor en la sociedad.

En este escenario, la alfabetización científica, entendida como "la capacidad de involucrarse en temas relacionados con la ciencia y con las ideas de la ciencia, como ciudadanos(as) reflexivos(as)" (OCDE 2017) adquiere un papel especial (Martín Páez et al 2019). Para promover esta alfabetización, las investigaciones en didáctica de las ciencias consideran que su enseñanza debe desarrollarse sobre la base de tres dimensiones competenciales: conceptual (abarcando el conocimiento de las leyes que rigen los fenómenos de la naturaleza), procedimental (considerando las distintas formas como los científicos trabajan) y actitudinal (National Research Council 1996, 2000). Según Greca y Meneses (2018), el desarrollo de las competencias científicas procedimentales y actitudinales se potencian con el uso de metodologías centradas en la figura del estudiante, trabajando a partir de situaciones problematizadoras con una práctica similar a la científica, como son por ejemplo la metodología de la indagación y del diseño ingenieril.

El presente trabajo se inserta en esta realidad, al describir y analizar un conjunto de secuencias didácticas indagatorias desarrolladas durante un trimestre lectivo en una clase de estudiantes de cuarto curso de primaria de un centro público de la capital de la provincia de Burgos - España, con el objetivo de identificar si las competencias asociadas con la práctica científica y las actitudes hacia las ciencias son potenciadas con el uso de un abordaje indagatorio. Concretamente pretendemos responder a la siguiente pregunta de investigación: “¿En qué medida el uso de la metodología de indagación y la del diseño ingenieril en las clases de Ciencias de la Naturaleza de Educación Primaria mejora las actitudes y emociones de los estudiantes hacia la Ciencia y contribuye a desarrollar algunas de las competencias asociadas con la práctica científica?"

\section{Antecedentes teóricos}

Aunque el enfoque competencial ha estado presente durante algunas décadas en las pautas educativas de la Unión Europea, Ortiz-Revilla et al. (2018) señalan que tanto el concepto de competencia como su implementación efectiva en el sistema educativo europeo en general, y específicamente en España, todavía representan un desafío a superar.

Cuando se trata de ciencia, el concepto de competencia científica ha evolucionado a lo largo de los años, y se manifiesta en varias definiciones complementarias (Laya y Martínez 2019). Por ejemplo, se puede asumir un concepto más directamente relacionado con la actividad profesional futura de los estudiantes como investigadores, es decir, considerando que las competencias "buscan formar a profesionales con amplios conocimientos y destrezas para emprender proyectos y programas de investigación de tal forma que puedan solucionar problemas" (García y Aguilar 2017), o un concepto más amplio, asociado con ciudadanos que en cualquier área de actividad puedan aplicar su conocimiento científico y comprensión de la naturaleza de la ciencia para tomar decisiones consistentes con las cuestiones socioambientales del presente siglo (OCDE 2017).

Además, hay varias clasificaciones de las competencias científicas en la literatura. García y Ladino (2008) presentan una tabla de competencias según su grado de complejidad, dividiendo las competencias científicas en básicas e investigativas. Franco (2015) separa 
la competencia científica en siete dimensiones: planteamiento de la investigación; manejo de la información; planificación y diseño de la investigación; recogida y procesamiento de datos; análisis de datos y emisión de conclusiones; comunicación de resultados; y actitud o reflexión crítica y trabajo en equipo. García y Aguilar (2017) utilizan tres criterios para definir las competencias científicas: indagación, experimentación y argumentación.

Independientemente de la clasificación considerada, se puede ver que todas tienen en común las características de trabajo de un científico. Por lo tanto, es razonable pensar que una buena metodología didáctica para la enseñanza de las ciencias que potencie el desarrollo de las competencias científicas debe proponer al estudiante actividades de aprendizaje que se asemejen en cierta medida a la práctica de un investigador o investigadora. La metodología de la indagación destaca en este escenario ya que es una "actividad más o menos abierta que introduce al alumno en el desarrollo de una serie de procedimientos asociados a la resolución de problemas, es decir, pequeñas investigaciones" (Harlen 1998). Y dentro de estas el enfoque de enseñanza por indagación basado en modelos (IBM) que permite potenciar la construcción y evaluación de modelos teóricos para explicar las evidencias disponibles (Steward et al., 2005; Schwarz \& Gwekwerere, 2007; Martínez-Chico et al., 2015) y donde los estudiantes que utilizan estas prácticas científicas de modelización y argumentación experimentan más compromiso y autoeficacia en su aprendizaje que con otras prácticas (Inkinen et al. 2020).

Además, cabe señalar que el aprendizaje basado en la indagación, al tener en cuenta los conocimientos previos del estudiante, promover la interacción sociocultural, el conflicto cognitivo y la regulación del aprendizaje, es coherente con las teorías socioconstructivistas de aprendizaje (Meneses y Caballero 2017). La indagación conforma un enfoque beneficioso para el aprendizaje de las ciencias, pero demanda un diseño cuidadoso de las actividades y del rol del profesor para conjugar un aprendizaje conceptual, procedimental y actitudinal (Rodríguez-Arteche, et al. 2019). En nuestra propuesta con el alumnado de educación primaria optamos inicialmente con indagaciones estructuradas y guiadas (Windschitl, 2003) donde se cuestionen sus concepciones sobre los fenómenos en estudio, diseñen experiencias para la búsqueda de pruebas con las que contrastarlas y cuestionarlas.

Finalmente, vale la pena señalar que el uso de actividades de investigación también contribuye al desarrollo de actitudes positivas hacia la ciencia, que deben enfatizarse en la educación primaria y secundaria, ya que en los últimos años ha habido un distanciamiento de la ciudadanía de las áreas científicas y un consecuente desapego del alumnado hacia las cuestiones científico-tecnológicas, como demuestran las investigaciones en el área (Toma et al 2019 Osborne y Dillon 2008, EC 2015).

\section{Las actividades indagatorias en los libros de texto de Ciencias}

Los libros de texto, como principal recurso pedagógico utilizado por los docentes (Rodríguez et al 2018, Park y Lavonen 2016), tienen un papel importante en la enseñanza pues dirigen la práctica profesional desde los aspectos específicos, como la elección de actividades docentes, hasta los más generales, como la implementación efectiva de los currículos oficiales.

Cuando se trata de la enseñanza de las ciencias, de acuerdo con las nuevas tendencias educativas, se espera que los docentes propongan actividades que permitan a los estudiantes desarrollar competencias y habilidades científicas, pudiendo construir ideas y fundamentos sobre los fenómenos de la naturaleza a través de estrategias y técnicas consistentes con la práctica científica. 
Sin embargo, como señalan varias investigaciones (Martínez y García 2003, López y García 2013, Pérez y Meneses 2019, Pastor y Limiñana 2019), los libros han evolucionado poco en los últimos quince años, al menos en el contexto español, se ha detectado un aumento en el contenido sobre historia del pensamiento evolutivo pero se mantiene la presencia de concepciones alternativas con escasas posibilidades de confrontase entre sí, y se han incorporado unas pocas actividades que potencian el desarrollo de procedimientos de carácter investigativo relacionados con la indagación y la resolución de problemas. En el contexto de la educación primaria, las actividades que figuran en los libros de texto de ciencia en español actuales, aunque se han aumentado su número y el de figuras, siguen siendo muy similares a las propuestas hace décadas. Por lo tanto, es necesario incorporar nuevas actividades para que realmente contribuyan al desarrollo de las competencias científicas (Laya y Martínez 2019).

\section{Los instrumentos para medir la evolución de los estudiantes}

Para la evaluación de las competencias científicas varios autores proponen rúbricas basadas en diferentes dimensiones (Ladino 2004; García y Ladino 2008, Franco-Mariscal 2015, Greca 2017, Greca y Ortiz 2018, García y Aguilar, 2017). Nosotros decidimos elaborar y utilizar un instrumento propio, adaptado del New Practical Test Assessment Inventory (Tamir et al., 1982) y del NPTAI (Ferrés et al., 2015), que contempla varias de estas dimensiones y que puede aplicarse en el contexto de la educación primaria. Dividimos los elementos del proceso de investigación en cinco competencias científicas: a) identificación y enunciado de preguntas científicamente orientadas, b) formulación de las ideas de los estudiantes como respuestas tentativas a las preguntas planteadas a modo de conjeturas o hipótesis, c) planificación y realización de diseños experimentales, d) capacidad para vincular explicaciones de las experiencias al conocimiento científico y e) saber resumir, comunicar y argumentar los resultados y el proceso indagatorio. En el Anexo A se presenta nuestra rúbrica para analizar las competencias científicas anteriores y los indicadores que hemos considerado.

En cuanto a la evaluación de las actitudes hacia la ciencia y las clases de ciencias, aunque en las últimas décadas se han desarrollado varios instrumentos (Wang y Berlin 2010, Zhang y Campbell 2011, Guzey et al. 2014, Summers y Khalick 2018) todos ellos, confeccionados en inglés, son muy extensos y poco adecuados para su uso en la educación primaria española. Uno de los pocos instrumentos que cumple con los criterios, brevedad en su aplicación y adaptado para niños y niñas de 6 a 12 años, es la escala diseñada y validada por Toma y Meneses (2019), procedente de la traducción al español del School Science Attitude Survey - SSAS (Kennedy et al. 2016). Se trata de una escala de actitudes hacia la ciencia a través de medidas de un único ítem, que mide seis constructos a través de diez cuestiones, de los cuales cuatro son unidimensionales y dos multidimensionales.

Por último, las emociones que los estudiantes sienten durante el proceso de aprendizaje pueden interferir tanto positiva como negativamente con su desempeño. Instrumentos para medir tales emociones comenzaron a desarrollarse desde la década de 1950 (Mandler y Sarason 1952) y han evolucionado para considerar diversas emociones, cada una con sus respectivos constructos, así como diferentes entornos de aprendizaje. Por ejemplo, Pekrun et al. (2011) desarrollaron el instrumento Achievement Emotions Questionmaire (AEQ) para medir nueve emociones (disfrute, esperanza, orgullo, alivio, ira, ansiedad, desesperanza, vergüenza y aburrimiento), relacionándolas con diferentes entornos (aula, examen) y situaciones de aprendizaje.

Nosotros hemos utilizado el cuestionario del Anexo B para analizar las actitudes y emociones de los estudiantes hacia la ciencia, basado en los instrumentos SSAS y AEQ. Consta de 
veinte ítems, diez tomados del SSAS para evaluar las actitudes hacia la ciencia (ítems 1, 4, $5,7,9,11,14,17,19,20)$ y otros diez del AEQ, cinco para medir las emociones positivas (ítems $2,6,8,12,15$ ) y otros cinco (ítems $3,10,13,16,18$ ) para las negativas.

\section{Diseño}

En este estudio ha participado una clase de cuarto curso de primaria de un centro público de la capital de la provincia de Burgos, con un total de 22 alumnos. Durante el último trimestre de del año escolar 2018-2019, aproximadamente la mitad de las clases de ciencias fueron desarrolladas con la metodología de indagación y de diseño ingenieril. Las temáticas abordadas fueron: mezclas y separación de sustancias (6 horas), propiedades de la luz y colores (12 horas), circuitos eléctricos y sus componentes (10 horas). En la Tabla 1 presentamos la denominación de la indagación o proyecto ingenieril, los contenidos científicos trabajados y los enlaces web donde se pueden consultar las actividades propuestas.

Tabla 1. Actividades desarrolladas

\begin{tabular}{|l|l|l|}
\hline Título & Contenidos trabajados & Enlace web \\
\hline $\begin{array}{l}\text { Vacaciones en } \\
\text { Barcelona }\end{array}$ & $\begin{array}{l}\text { La materia y sus cambios. Sustancias } \\
\text { puras y mezclas. Mezclas homogéneas y } \\
\text { heterogéneas. Métodos de separación de } \\
\text { mezclas. }\end{array}$ & $\begin{array}{l}\text { http://www.webciencia.es/index. } \\
\text { php/articulos/242-vacaciones-en- } \\
\text { barcelona-fases-e-informacion }\end{array}$ \\
\hline $\begin{array}{l}\text { El cumpleaños } \\
\text { de Sofia }\end{array}$ & $\begin{array}{l}\text { Comportamiento de los cuerpos ante la } \\
\text { luz y de la luz ante los objetos (reflexión } \\
\text { y refracción). Descomposición de la luz } \\
\text { blanca. }\end{array}$ & $\begin{array}{l}\text { http://www.webciencia.es/ } \\
\text { index.php/articulos/250-el- } \\
\text { cumpleanos-de-sofia-fases-e- } \\
\text { informacion }\end{array}$ \\
\hline $\begin{array}{l}\text { Jugando con } \\
\text { luces }\end{array}$ & $\begin{array}{l}\text { El color y mezclas de colores. Identifica- } \\
\text { ción de los colores básicos. Descomposi- } \\
\text { ción de la luz blanca. }\end{array}$ & $\begin{array}{l}\text { http://www.webciencia.es/index. } \\
\text { php/articulos/256-jugando-con- } \\
\text { luces-fases-e-informacion }\end{array}$ \\
\hline $\begin{array}{l}\text { Jugando con } \\
\text { el color de los } \\
\text { objetos }\end{array}$ & $\begin{array}{l}\text { El color de los objetos. } \\
\text { http://www.webciencia.es/index. } \\
\text { php/articulos/257-jugando-con- } \\
\text { el-color-de-los-objetos-fases-e- } \\
\text { informacion }\end{array}$ \\
\hline $\begin{array}{l}\text { Electricidad } \\
\text { en la casita }\end{array}$ & $\begin{array}{l}\text { Clasificación de materiales por su capa- } \\
\text { cidad de conducción: aislantes y conduc- } \\
\text { tores de la electricidad. Electricidad y } \\
\text { corriente eléctrica. Circuitos eléctricos: } \\
\text { elementos. }\end{array}$ & $\begin{array}{l}\text { http://www.webciencia.es/index. } \\
\text { php/articulos/articulos/275- } \\
\text { electricidad-en-la-casita-fases-e- } \\
\text { informacion }\end{array}$ \\
\hline $\begin{array}{l}\text { Conecta" } \\
\text { instrón }\end{array}$ & $\begin{array}{l}\text { Electricidad. La corriente eléctrica. Con- } \\
\text { sus elementos). Construcción de un juego } \\
\text { eléctrico de preguntas y respuestas. }\end{array}$ & $\begin{array}{l}\text { http://www.webciencia.es/ } \\
\text { index.php/metodo-ingenieril- } \\
\text { 2/281-construccion-de-un-juego- } \\
\text { electrico-de-unir-conceptos- } \\
\text { fases-e-informacion }\end{array}$ \\
\hline
\end{tabular}

Para analizar la evolución de las competencias procedimentales asociadas con la práctica científica, durante y después de la aplicación de la propuesta didáctica, los materiales producidos por los equipos durante las actividades didácticas fueron clasificados según la rúbrica del Anexo $A$. Además, después de la enseñanza los estudiantes contestaron a las cinco preguntas del cuestionario del Anexo $C$, el cual permitió evaluar el nivel competencial de las competencias científica Cl (pregunta 1), Cll (pregunta 4), CIII (preguntas 2 y 3 ) y CIV (pregunta 5) 
Para el análisis de la evolución de las actitudes hacia las ciencias y emociones, los estudiantes contestaron el cuestionario del Anexo B antes y después del desarrollo de la propuesta didáctica.

Se empleó el paquete estadístico SPSS v.25 para analizar los resultados. Dado el tamaño muestral, y debido a que la prueba de Shapiro Wilks mostró que los datos no siguen una distribución normal $(p>0.05)$ se usó estadística no paramétrica. Específicamente, se empleó el test de Wilcoxon para comprobar en qué medida los resultados del postest difieren significativamente de los del pretest, y la prueba $U$ de Mann Whitney para realizar comparaciones entre los resultados de los chicos y las chicas. En ambos casos, se estableció un valor de alfa de $p<0.05$

\section{Las unidades didácticas desarrolladas}

A modo de ejemplo presentamos, en primer lugar, una de las cinco indagaciones llevadas a cabo con los estudiantes, sobre la interacción de la luz con la materia y, en segundo lugar, un proyecto de ingeniería donde se propone el diseño y construcción del juego "Conecta".

Primer ejemplo. Utilizando la metodología didáctica indagatoria, propusimos a los estudiantes estudiar que le ocurre a la luz cuando interacciona con la materia. Para ello, seguimos un ciclo formado por cinco etapas: i) situación problematizadora, ii) problema de investigación e hipótesis, iii) diseño experimental, iv) resultados y conclusiones, y v) afianzamiento y comunicación. Estas etapas son flexibles y dependiendo de las circunstancias propias que se producen durante la implementación, habitualmente se producen conexiones entre ellas (Pérez y Meneses, 2019).

En la presente indagación utilizamos como situación problematizadora el siguiente relato, que es leído por todos los estudiantes: "El sábado pasado fue el cumpleaños de Sofía. Sus padres prepararon una fiesta sorpresa en una cafetería que tenía un local grande con muchos juegos para niños. Los amigos de Sofía se divirtieron en la piscina de bolas, en el pula-pula y en el tobogán. También recibieron una ficha cada uno para utilizarla en una máquina que al azar proporcionaba regalos. Sofía consiguió unas gafas de lentes rojas, su amiga Clara un juguete para hacer burbujas de jabón, Gabriel una linterna de luz blanca, Pedro una lupa, Rosa un espejito, etc. Todos los niños obtuvieron su regalo. Jugando con los objetos que salieron de la máquina se dieron cuenta de varias cosas que llamaron la atención; por ejemplo, con las gafas de Sofía las bolas de la piscina cambiaban de color; cuando la linterna de Gabriel iluminaba las burbujas de jabón se veían de muchos colores, pareciendo un arcoíris; con la lupa de Pedro se veían los objetos más grandes; mirando al espejo veían sus caras, cuando chocaba la luz de la linterna con el espejo el haz de luz rebotaba, sin embargo, si chocaba con la pared negra no, etc."

Mediante un debate promovido por el profesor se analiza el relato, se intercambian ideas y entre todos se identifican las preguntas más susceptibles de investigación; durante la aplicación de la propuesta, por ejemplo, entre otras se formularon: “¿por qué cuando la luz de la linterna choca con el espejo rebota?", "¿por qué cuando la luz de la linterna llega a las pompas aparecen colores como los del arco-iris?", "¿por qué se ven con la lupa los objetos más grandes?" Al contestar a estas y otras preguntas los estudiantes expresaban sus ideas previas: "el color de los objetos cambia según sea la luz", "cuando la luz da a una camiseta de lentejuelas se refleja", "si teñimos una goma de borrar blanca de color azul es azul, pero su color sigue siendo blanco", etc.

Antes de comenzar la segunda etapa, el profesor dirige el debate con los estudiantes para proponer el problema de investigación que definitivamente se va a abordar:"¿cómo se 
comportan distintos materiales o sustancias cuando incide la luz sobre ellos?" Como respuesta al mismo los estudiantes emiten, en colaboración con el profesor, sus hipótesis:

- la luz traspasa a algunos objetos, como los cristales y algunos plásticos.

- hay objetos en los cuales la luz rebota, por ejemplo, en un espejo o en el agua en reposo.

- la luz puede quedarse en algunos objetos, es decir, no traspasa ni rebota en el objeto, o traspasa parcialmente.

En la tercera etapa, los estudiantes debaten en grupo y presentan propuestas de diseños experimentales para testar las hipótesis planteadas. Proponen, por ejemplo, utilizar un espejo y una linterna. El experimento puede ser realizado iluminando diferentes sustancias con una linterna que emite luz blanca, o bien, empleando un equipo sencillo de óptica. Antes de comenzar el experimento, se les propone que escriban en una tabla sus predicciones sobre lo que creen que va a ocurrir cuando se iluminan diferentes materiales con luz blanca.

Tras ejecutar los experimentos, los estudiantes recogen en otra tabla lo que observan, ayudándose de un dibujo, y lo comparan con sus predicciones. Estos datos son utilizados para contrastar las hipótesis planteadas. El profesor presenta los términos refracción y reflexión, explica los tipos de materiales (opacos, transparentes y traslúcidos) y los grupos los clasifican experimentando.

En la última etapa, de afianzamiento y comunicación, los estudiantes clasifican nuevos materiales, elaboran un mapa conceptual, reflexionan sobre el proceso que han seguido para contestar al problema y lo comunican al resto de compañeros.

Segundo ejemplo. Para implementar la metodología que seguimos en el método ingenieril, propusimos a los estudiantes que diseñaran y construyeran un juego con preguntas y respuestas donde se encendiera una bombillita cuando se contestaran correctamente las preguntas. Pretendemos que desempeñen el papel de ingenieros proyectando y elaborando un producto que responda a la necesidad planteada. La validación y utilidad del producto desarrollado están vinculadas a los condicionantes establecidos, como: costo, material disponible, requisitos, estética, calidad, entre otros (Pérez y Meneses, 2019). De manera similar a la propuesta desarrollada en la indagación científica, en el método ingenieril proponemos seguir cinco etapas, también intercambiables según las circunstancias: i) definición del problema, ii) búsqueda de información pertinente, iii) planificación y diseño del prototipo, iv) prueba del prototipo, v) mejora del producto.

Dado que los alumnos ya sabían cómo realizar un circuito eléctrico para que brille una bombilla, pues antes habían realizado una indagación sobre los mismos, se les propuso el reto de construir un juego eléctrico de preguntas y respuestas, conocido como el clásico "Conecta" o "Lectron" (análogo al de la Figura 1), con tres condiciones a) el juego tendrá, al menos, ocho preguntas y sus correspondientes respuestas; b) debe encenderse una bombilla solo cuando se conteste de forma correcta a las preguntas; y c) la instalación eléctrica apenas debe verse. Las preguntas y respuestas deben ser formuladas por los estudiantes, seleccionando una temática de su interés. Se valorará también la estética del juego.

Se establecen dos limitaciones a los estudiantes. Sólo pueden utilizar los materiales proporcionados por el profesor (cartón duro, fundas de plástico transparentes, chinchetas, encuadernadores, hojas de papel, cables, bombillas, portalámparas, pilas) y, una vez realizada la planificación y el diseño del producto, disponen de 40 minutos como máximo para construir su prototipo del juego Conecta. 


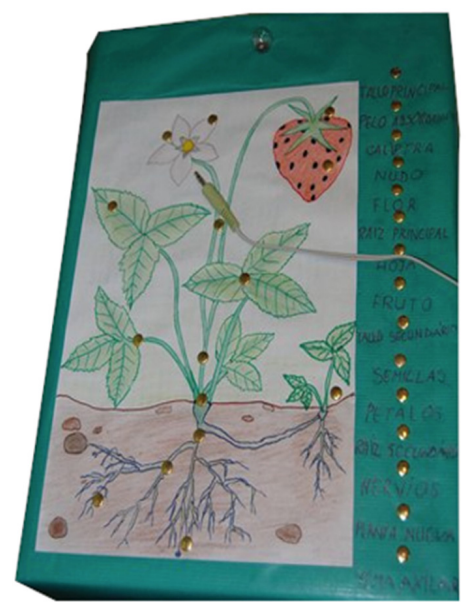

Figura 1. Ejemplo de juego a ser ejecutado.

Antes de comenzar la etapa de planificación y diseño del prototipo, el profesor formula preguntas en el aula tratando de indagar sobre las ideas previas que tienen los alumnos acerca de un circuito eléctrico. En la tercera etapa, los distintos grupos de alumnos primero piensan en el circuito y dibujan un esquema del mismo. El profesor puede ayudar realizando un dibujo análogo a los de la Figuras 2 , sin los cables que unen preguntas y respuestas, preguntándoles sobre cómo los conectarían para que la bombilla solo se encendiera cuando se contesta correctamente.
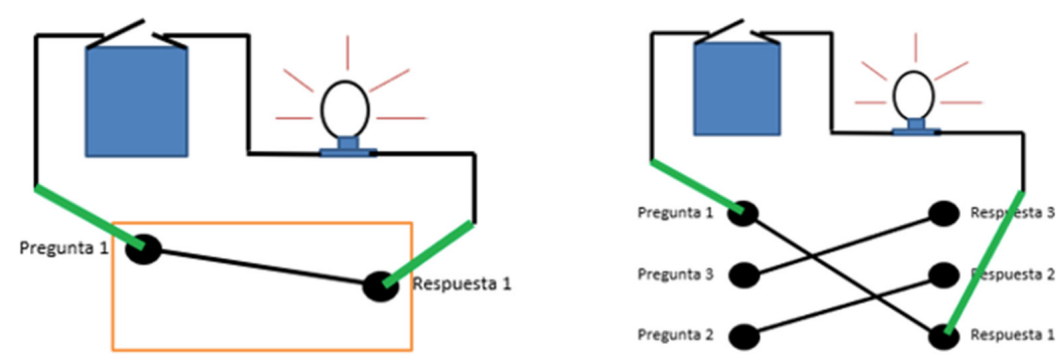

Figura 2. Ejemplo de dibujos del circuito a ser ejecutado.

Una vez encontrada la solución eléctrica, los grupos realizan un diseño del juego para que todos los componentes que lo conforman queden perfectamente fijos y con un aspecto visual agradable (Figura 3).

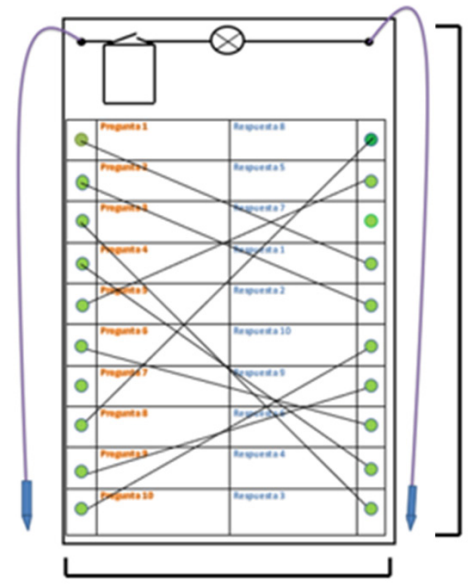

Figura 3. Diseño del juego en un soporte de madera o cartón. 
En la cuarta etapa los equipos tienen que construir su prototipo, siguiendo los pasos definidos en la fase anterior. Al final, tras presentar sus prototipos a los compañeros de clase, los grupos pueden mejorar su juego, corrigiendo lo que sea necesario, probando y presentando a los compañeros y compañeras de la clase el juego definitivo, quienes valoran su calidad atendiendo a los criterios establecidos.

\section{Resultados y su análisis}

\section{Competencias científicas}

En la Tabla 2 se presentan los resultados sobre la evolución experimentada por el alumnado en las dimensiones competenciales consideradas, según la rúbrica del anexo $\mathrm{A}$, aplicada en las cinco indagaciones llevadas a cabo en el aula. Las columnas 2 a 5 muestran el nivel competencial alcanzado por los cinco grupos en las cuatro primeras competencias de la rúbrica, que se analizaron a través de las fichas de las indagaciones elaboradas por los grupos (estas fichas se puedan consultar en las páginas web indicadas en la Tabla 1). La columna 6 muestra el nivel competencial alcanzado por el alumnado, establecido a través de las contestaciones individuales al cuestionario (anexo $\mathrm{C}$ ) después del desarrollo de las cinco indagaciones y el proyecto ingenieril.

En términos generales, de los resultados de la Tabla 2, se puede decir que los estudiantes después de la enseñanza recibida han llegado a un nivel razonable de desarrollo de dos competencias, identificar preguntas $(\mathrm{Cl})$ y analizar datos y elaborar conclusiones (CIV). Sin embargo, su capacidad para emitir hipótesis razonadas (CII) es limitada y la de planificar y realizar diseños experimentales se encuentra en un nivel incipiente (CIII).

Tabla 2 Evolución en el nivel competencial de los grupos y final alcanzado por los alumnos

\begin{tabular}{|c|c|c|c|c|c|c|}
\hline Competencia & $\begin{array}{l}\text { Sustancias } \\
\text { y mezclas }\end{array}$ & $\begin{array}{c}\text { Interacción } \\
\text { de luz y } \\
\text { materiales }\end{array}$ & $\begin{array}{l}\text { Mezcla de } \\
\text { colores }\end{array}$ & $\begin{array}{c}\text { Color de } \\
\text { los objetos }\end{array}$ & $\begin{array}{l}\text { Circuitos } \\
\text { eléctricos }\end{array}$ & $\begin{array}{c}\text { Cuestionario } \\
\text { final }\end{array}$ \\
\hline \multirow{5}{*}{$\mathrm{Cl}$} & N1: 0 & N1: 0 & N1: 0 & N1: 0 & N1: 0 & N1: 0 \\
\hline & N2: 2 & N2: 0 & N2: 0 & N2: 0 & N2: 1 & N2: 2 \\
\hline & N3: 0 & N3: 0 & N3: 4 & N3: 1 & N3: 0 & N3: 0 \\
\hline & N4: 0 & N4: 5 & N4: 0 & N4: 1 & N4: 4 & N4: 20 \\
\hline & N5: 3 & N5: 0 & N5: 1 & N5: 3 & N5: 0 & N5: 0 \\
\hline \multirow{5}{*}{ CII } & N1: 0 & N1: 0 & N1: 0 & N1: 0 & N1: 0 & N1: 1 \\
\hline & N2: 0 & N2: 0 & N2: 2 & N2: 0 & N2: 0 & N2: 3 \\
\hline & N3: 2 & N3: 1 & N3: 0 & N3: 2 & N3: 0 & N3: 17 \\
\hline & N4: 2 & N4: 1 & N4: 2 & N4: 3 & N4: 3 & N4: 1 \\
\hline & N5: 1 & N5: 3 & N5: 1 & N5: 0 & N5: 2 & N5: 0 \\
\hline \multirow{5}{*}{ CIII } & & N1: 0 & N1: 1 & N1: 1 & N1: 0 & N1: 9 \\
\hline & & N2: 3 & N2: 4 & N2: 3 & N2: 0 & N2: 6 \\
\hline & No trabajada & N3: 2 & N3: 0 & N3: 1 & N3: 5 & N3: 4 \\
\hline & & N4: 0 & N4: 0 & N4: 0 & N4: 0 & N4: 3 \\
\hline & & N5: 0 & N5: 0 & N5: 0 & N5: 0 & N5: 0 \\
\hline \multirow{5}{*}{ CIV } & N1: 0 & N1: 0 & N1: 0 & N1: 0 & N1: 0 & N1: 1 \\
\hline & N2: 0 & N2: 0 & N2: 0 & N2: 0 & N2: 1 & N2: 1 \\
\hline & N3: 0 & N3: 0 & N3: 0 & N3: 0 & N3: 0 & N3: 8 \\
\hline & N4: 5 & N4: 5 & N4: 4 & N4: 4 & N4: 4 & N4: 11 \\
\hline & N5: 0 & N5: 0 & N5: 1 & N5: 1 & N5: 0 & N5: 1 \\
\hline
\end{tabular}


Específicamente, sobre la competencia científica $\mathrm{Cl}$, identificar problemas de la situación problemática, los grupos evolucionaron con altibajos a lo largo de la propuesta didáctica, dependiendo de la situación problemática propuesta. Mientras que en la situación problemática inicial el $40 \%$ de los grupos plantearon cuestiones de nivel 2 (N2), resultado aceptable, aunque las preguntas no pudieran clasificarse como científicas, en la evaluación individual final (pregunta 1 del cuestionario Anexo C) una amplia mayoría de las preguntas identificadas por los alumnos se clasificaron en el nivel 4 (N4). El grupo 3, por ejemplo, en la primera actividad propone la siguiente pregunta de investigación: “¿cómo funciona la planta desalinizadora?", clasificada en el nivel 2, siendo poco científica; ya en la cuarta actividad, los mistos estudiantes proponen: "¿por qué se forman otros colores cuando se juntan dos colores?", clasificada con el nivel 4 , pues no deja claro la variable dependiente. Sin embargo, fueron pocas las situaciones en que los estudiantes (en grupo o individualmente) formularon preguntas de nivel 5 , lo que cabía esperar pues no se debatió durante la propuesta sobre el concepto de variables (dependientes y/o independientes). Un ejemplo de pregunta clasificada con el nivel 5 fue la propuesta por el equipo 3: “¿cómo se puede generar la electricidad para que brille la bombilla?".

Respecto a la competencia científica Cll, formular hipótesis, en general se pudo percibir una ligera evolución a lo largo del desarrollo de la propuesta didáctica, de tal forma que en la última secuencia todos los equipos fueron clasificados con los niveles N4 y N5. Sin embargo, cuando se aplicó el cuestionario final individual (cuestión 4, Anexo C), con una pregunta de investigación un poco compleja ("¿Cómo se puede conseguir que el brillo de una bombilla cambie?"), la mayoría de los estudiantes formuló hipótesis que se clasificaron en el nivel N3, pudiendo identificar variables que interfieren con el brillo de la lámpara, pero sin proponer relaciones causales, como por ejemplo "cuanto más voltaje tiene la pila, más brillará la bombilla". Entendemos que este tipo de capacidad o no se puede desarrollar en estudiantes de nueve años, o debe trabajarse mejor en futuras propuestas didácticas.

Debido a que los estudiantes no entienden los conceptos de variables dependiente e independientes y tienen dificultades para construir relaciones causales, su capacidad para planificar un diseño experimental capaz de contrastar las hipótesis se ve comprometida. Además, su experiencia en realizar diseños o planificaciones es prácticamente inexistente pues muy pocas veces se trabaja esta capacidad en el aula. Así que, a lo largo de la propuesta didáctica la evolución respecto a la tercera competencia científica, planificar o realizar el diseño experimental (CIII), fue pequeña pues ningún equipo pudo evolucionar a los niveles N4 o N5. En el cuestionario final (cuestiones 2 y 3, Anexo C), donde las dificultades se manifiestan individualmente, nueve estudiantes (aproximadamente el $40 \%$ de la clase) aún confundieron el diseño experimental con la formulación de hipótesis ("si estiras mucho de la goma más lejos llegará el garbanzo, si estiras menos más cerca quedará") y seis (aproximadamente el $27 \%$ de la clase) sólo fueron capaces de presentar algunos de los materiales apropiados para el experimento ("cambiando de goma a una más estirable, o más fuerte, o más gorda, o más fina etc"). Aun así, se puede percibir una ligera evolución, ya que tres estudiantes (14\%) fueron capaces de proponer un experimento del nivel N4 ("tiro varios garbanzos estirando la goma más o menos y mido lo que se alarga la goma con una regla o poniendo rayas y también hasta donde llega").

Desde la primera situación problematizadora, los estudiantes presentaron dominio en la competencia científica CIV, analizar datos y elaborar conclusiones argumentadas, siendo casi todas las actividades realizadas por los alumnos en esta actividad inicial clasificadas con el nivel 4, un buen dominio, y este comportamiento se mantuvo a lo largo de la aplicación de la propuesta. Por ejemplo, sobre el proceso de reflexión de la luz, tras realizar los experimentos de interacción de la luz con los materiales, uno de los grupos escribió: "en un espejo la luz rebota dependiendo de la posición", evidenciando una comprensión 
preliminar de la ley de reflexión de la luz. Otro ejemplo de conclusión presentada fue "cuando un haz de luz blanca incide sobre un objeto colorido lo vemos del color del pigmento que tenga, si lanzas luz roja sobre un objeto colorido, dependiendo del pigmento lo vemos de un color u otro". En el cuestionario final (cuestión 5, Anexo C), la mitad de los estudiantes obtuvieron el nivel N4, ocho (40\%) emitieron conclusiones clasificadas como N3, un estudiante (5\%) no escribió nada, y otro (5\%) obtuvo con su explicación el nivel máximo: "si tiene más temperatura estaría liquido; si tiene menos temperatura estaría sólido; si la temperatura es cero, estaría sólido y líquido".

Aunque se ha solicitado en varios momentos de la aplicación de la propuesta que los alumnos escribieran y hablaran de sus observaciones y conclusiones, no fue realizado un trabajo formal de comunicación y argumentación. Por ello, decidimos no analizar la competencia científica CV.

\section{Actitudes hacia las clases de ciencias y emociones}

En la Tabla 3 se presentan los resultados obtenidos de las contestaciones de los estudiantes al cuestionario sobre actitudes y emociones, antes y después del proceso de enseñanza. Tras la intervención, los estudiantes han manifestado un mayor disfrute durante las clases de ciencias, que tal vez se deba a una menor dificultad percibida de estas clases. También ha sido mayor la media del constructo asociado con la relevancia de la ciencia para su vida personal. Finalmente, hubo una mayor cantidad de emociones positivas. La intervención no tuvo un impacto significativo en el resto de las variables estudiadas.

Tabla 3 Resultado de la aplicación de la escala sobre actitudes hacia la ciencia y emociones

\begin{tabular}{|c|c|c|c|c|c|}
\hline \multirow{2}{*}{ Constructo } & \multicolumn{2}{|c|}{ Pretest } & \multicolumn{2}{|c|}{ Postest } & \multirow{2}{*}{$\begin{array}{c}\text { Valor } \\
\mathbf{p}\end{array}$} \\
\hline & $\mathrm{M}$ & SD & $\mathrm{M}$ & SD & \\
\hline Intenciones (ítem 1) & 3,45 & 1,05 & 3,45 & 1,05 & .923 \\
\hline Disfrute durante las clases (ítem 4) & 4,05 & 1,05 & 4,77 & 0,43 & .008 \\
\hline Relevancia personal (ítem 5, 19, 20) & 4,00 & 0,87 & 4,41 & 0,70 & .034 \\
\hline Relevancia social (ítem 17) & 4,50 & 0,83 & 4,73 & 0,63 & .305 \\
\hline Utilidad (ítem 11, 14) & 4,20 & 0,85 & 4,39 & 0,79 & .583 \\
\hline Dificultad (ítem 7) & 2,90 & 1,77 & 1,73 & 1,03 & .007 \\
\hline Autoeficacia (ítem 9) & 3,40 & 0,99 & 3,41 & 1,10 & .523 \\
\hline Emociones negativas (ítems $3,10,13,16,18$ ) & 1,78 & 0,62 & 1,61 & 0,66 & .325 \\
\hline Emociones positivas (ítems 2, 6, 8, 12, 15) & 3,64 & 0,83 & 4,28 & 0,60 & .001 \\
\hline
\end{tabular}

Tabla 4. Evolución de las actitudes hacia las ciencias y emociones

\begin{tabular}{|l|c|c|c|c|}
\hline Constructo & Disminuyen & Aumentan & Igual & Total \\
\hline Intenciones & 7 & 7 & 4 & 18 \\
\hline Disfrute de las clases & 1 & 10 & 9 & 20 \\
\hline Relevancia personal & 3 & 11 & 6 & 20 \\
\hline Relevancia social & 2 & 6 & 12 & 20 \\
\hline Utilidad & 6 & 10 & 4 & 20 \\
\hline Dificultad & 12 & 3 & 5 & 20 \\
\hline Autoeficacia & 5 & 8 & 7 & 20 \\
\hline Emociones negativas & 11 & 7 & 2 & 20 \\
\hline Emociones positivas & 1 & 14 & 4 & 19 \\
\hline
\end{tabular}


En la Tabla 4 presentamos los resultados sobre el número de estudiantes que disminuyeron, aumentaron o mantuvieron las actitudes o emociones después de aplicar las cinco secuencias de indagación y el proceso ingenieril sobre el Producto 'Conectar'.

Algo interesante a considerar es que, si se analiza el impacto de la intervención controlando la variable sexo de los participantes, los resultados cambian, de modo que son aplicables: solo a chicas para las variables disfrute; solo a chicos para las variables relevancia personal y dificultad; a ambos sexos para la variable emociones positivas. En consecuencia, la intervención parece que ha sido eficaz en mejorar, por un lado, las variables relevancia personal, dificultad y emociones positivas en los chicos y, por otro las variables: disfrute en las clases de ciencias y emociones positivas en las chicas.

\section{Comentarios finales y conclusiones}

El currículo básico de la educación primaria española (BOE 2014) establece que en esta etapa los estudiantes deben iniciarse en el desarrollo de las competencias asociadas con la formación de ciudadanos y ciudadanas que, en cualquier área de actividad, puedan aplicar su conocimiento científico y comprensión de la naturaleza de la ciencia para tomar decisiones consistentes con las cuestiones socioambientales del presente siglo (OCDE 2017, Fraiha et al. 2018).

En la literatura de enseñanza de las ciencias, se pueden encontrar ejemplos de aplicación y evaluación de propuestas didácticas que utilizan actividades indagativas como herramienta para potenciar el desarrollo de estas competencias. Sin embargo, existen pocos estudios sobre el impacto de estas propuestas en la evolución de las competencias científicas de los estudiantes durante un período de tiempo más largo. Además, cuando se trata del uso del diseño ingenieril, los estudios son prácticamente inexistentes.

En este artículo, presentamos el análisis de un estudio desarrollado durante un trimestre académico con una clase de cuarto curso de primaria, donde fueron utilizados los dos enfoques didácticos mencionados. Se utilizaron cinco situaciones problematizadoras para trabajar contenidos conceptuales de tópicos de la física, así como procedimentales y actitudinales, mediante un enfoque de indagación. Además, como proyecto final del trimestre, los estudiantes realizaron un prototipo de juego por medio del enfoque de diseño ingenieril. Aunque este estudio ha aportado datos y potencialidades del alumnado de interés, señalamos dos limitaciones que es preciso destacar; por una parte, el reducido tamaño de la muestra y el corto periodo de implementación implica que las conclusiones sean muy preliminares; y, por otra parte, la utilización de la rúbrica ad hoc del Anexo A para medir las competencias científicas requiere de un estudio para valorar su validez y fiabilidad; aunque ha sido adaptada de rúbricas utilizadas en el ámbito de la educación secundaria, posiblemente requiera de ajustes en los indicadores o niveles para mejorar la valoración de las competencias,

En general, fue posible concluir que, aunque se espera que los estudiantes aprendan competencias científicas utilizando estos enfoques, es necesario considerar su desarrollo cognitivo en las diferentes etapas de la educación formal al plantear las actividades. Así, a partir del análisis de los resultados, por ejemplo, hay indicios de que a la edad considerada en el estudio (9-10 años), los estudiantes tienen dificultad para construir relaciones causales, lo que restringe su desarrollo de competencias científicas a ciertos límites. Estas dificultades afectan a la discusión de los conceptos de variables dependientes e independientes, clave para una buena formulación de hipótesis y planteamiento de diseños experimentales. Por lo tanto, considerar y hacer evolucionar el desarrollo cognitivo de los estudiantes en las diferentes etapas de la educación formal debería ser la columna vertebral en la 
planificación de propuestas didácticas que busquen mejorar la evolución de las competencias científicas. Para ello, se necesitan establecer debates y realizar estudios focalizados a cómo implementar los procesos indagatorios en el aula de educación primaria que permitan contestar a las preguntas: cómo ayudar al alumnado a diferenciar hipótesis y predicciones o a establecer diseños experimentales para contrastar sus hipótesis, cómo asesorarles en los experimentos que haya que controlar variables, cómo relacionar las pruebas con la teoría, cómo desarrollar modelos explicativos, entre otras.

Además, es importante señalar la importancia de elegir buenas situaciones problemáticas, que faciliten el proceso de formulación de preguntas y emisión de hipótesis. Al analizar la evolución de las competencias durante la aplicación las propuestas algunas temáticas, posiblemente por estar más contextualizadas o ser más del interés del alumnado, propiciaron en el mismo una discusión más rica. Lo cual indica la necesidad de investigar cuáles son las problemáticas que interesan y motivan al alumnado, cómo presentarlas, qué recursos utilizar, para que generen ideas y cuestiones científicas.

\section{Referencias bibliográficas}

BOE (2014). R.D. 126/2014, de 28 de febrero, por el que se establece el currículo básico de la Educación Primaria

European Commision (2015). Science education for Responsible Citizenship [versión PDF]. DOI: https://doi.org/10.2777/12626

Fraiha, S., Paschoal, W., Perez, S., Tabosa, C. y Silva, C. R. (2018). Atividades indagativas e o desenvolvimento de habilidades e competências: um relato de experiencia no curso de Física da Universidade Federal do Pará. Revista Brasileira de Ensino de Física, 40 (4) e4403.

Franco Mariscal, A. (2015). Competencias científicas en la enseñanza y el aprendizaje por investigación. Un estudio de caso sobre corrosión de metales en secundaria. Enseñanza de las Ciencias, 32 (2) 231-252. https://ensciencias.uab.es/article/view/ v33-n2-franco

Ferrés, C., Marbà, A. y Sanmartí, N. (2015). Trabajos de indagación de los alumnos: Instrumentos de evaluación e identificación de dificultades. Revista Eureka sobre Enseñanza y Divulgación de las Ciencias, 12(1), pp. 22-37. https://revistas.uca.es/ index.php/eureka/article/view/2900

García Contreras, G. A. y Ladino Ospina, Y. (2008). Desarrollo de competencias científicas a través de una estrategia de enseñanza y aprendizaje por investigación. Studiositas, 3 (3) 7-16.

Greca I. y Meneses J. A. (2018). Proyectos STEAM en la educación primaria: fundamentos y aplicaciones prácticas. Madrid: Dextra.

Guzey SS, Harwell M, Moore T. (2014). Development of an instrument to assess attitudes toward STEM. School Science and Mathematics, 114, 271. https://doi.org/10.1111/ ssm.12077

Harlen, W. (1998). Enseñanza y aprendizaje de las ciencias. Madrid: Morata.

Inkinen, J., Klager, C., Juuti, K., Schneider, B., Salmela-Aro, K., Krajcik, J. y Lavonen, J. (2020). High school students' situational engagement associated with scientific practices in designed science learning situations. Science Education, 104(4), 667-692. DOI: https://doi.org/10.1002/sce.21570 
Kennedy, J., Quinn, F. y Taylor, N. (2016). The school science attitude survey: a new instrument for measuring attitudes towards school science. International Journal of Research Method Education, 39, 422-445. DOI: https://doi.org/10.1080/17437 27X.2016.1160046

Ladino Ospina, Y. (2004). Una propuesta de evaluación de competencias en química general. Tesis en educación. Universidad Pedagógica Nacional. Bogota, Colombia.

Laya Iglesias, P. y Martínez Losada, C. (2019). La competencia científica en los libros de texto de Educación Primaria. Ápice. Revista de Educación Científica, 3(1), 71-83. DOI: https://doi.org/10.17979/arec.2019.3.1.5000

López-Valentin, D. M. y Guerra-Ramos, M. T. (2013). Análisis de las actividades de aprendizaje incluidas en libros de texto de ciencias naturales para educación primaria utilizados en México. Enseñanza de las Ciencias, 31 (2), 173-191

Mandler, G. y Sarason, S. B. (1952) A study of anxiety and learning. Journal of Abnormal and Social Psychology, 47(2), 166-173

Martínez-Chico, M., Jiménez-Liso, M.R. y López-Gay, R. (2015). Efecto de un programa formativo para enseñar ciencias por indagación basada en modelos, en las concepciones didácticas de los futuros maestros. Revista Eureka sobre Enseñanza y Divulgación de las Ciencias, 12(1), 149-166. https://revistas.uca.es/index.php/ eureka/article/view/2908

Martínez Losada, C. y García Barros, S. (2013). Las actividades de primaria y ESO incluidas en libros escolares: ¿qué objetivo persiguen? ¿Qué procedimientos enseñan? Enseñanza de las Ciencias 21 (2), 243-264.

Martín-Páez, T., Carrillo-Rosúa, J., Lupiáñez-Gómez, J. L. y Vílchez-González, J. M. (2019). Análisis de las pruebas externas de evaluación de la competencia científico-tecnológica de 6. de Ed. Primaria en España (2016). Enseñanza de las ciencias, 37(2), 127-149. DOI: https://doi.org/10.5565/rev/ensciencias.2632

Meneses, J. A. y Caballero, C. (2017). La metodología indagatoria en educación primaria. Una mirada desde la perspectiva del aprendizaje significativo. $X$ Congreso Internacional sobre investigación en didáctica de las ciencias, Sevilla.

National Research Council (1996). The National Science Education Standards. Washington D.C.: The National Academies Press

National Research Council (2000). Inquiry and the national science education standards: A guide for teaching and learning. Washington, D. C.: The National Academies Press

OCDE (2017). PISA 2015 Science Framework. Assessment and Analytical Framework: Science, Reading, Mathematics, Financial Literacy and Collaborative Problem Solving. Oecd Publis- hing: Paris. DOI: https://doi.org/10.1787/97892642818203-en

Ortiz-Revilla, J., Greca, I. M. y Adúriz-Bravo, A. (2018). La Educación STEAM y el desarrollo competencial en la Educación Primaria. En I. M. Greca y J. A. Meneses Villagrá (Eds.), Proyectos STEAM para la Educación Primaria. Fundamentos y aplicaciones prácticas. Madrid: Dextra

Osborne, J. y Dillon, J. (2008). Science education in Europe: Critical reflections. Recuperado de: https://www.nuffieldfoundation.org/about/publications/science-education-ineurope-critical-reflections 
Pastor, A. y Limiñana Morcillo, R. (2019). La evolución en una serie temporal de libros de texto (1945-2016): Cambios en la metodología y en la presencia de concepciones alternativas. Ápice. Revista de Educación Científica, 3(2), 71-92. DOI: https://doi. org/10.17979/arec.2019.3.2.4811

Park, D. y Lavonen, J. (2013). An analysis of standards-based high school physics textbooks of Finland and the United States. In M. S. Khine (Ed.) Critical Analysis of Science Textbooks: Evaluating Instructional Effectiveness. Dordrecht, The Netherlands: Springer

Pérez, S y Meneses Villagrá, J. A. (2019). La competencia científica en las actividades de aprendizaje incluidas en los libros de texto de Ciencias de la Naturaleza, Revista Eureka sobre Enseñanza y Divulgación de las Ciencias, 17(2), 2101. DOI: https://doi. org/10.25267/Rev_Eureka_ensen_divulg_cienc.2020.v17.i2.2101

Pekrun, R., Goetz, T., Frenzel, A. C., Barchfeld, P. y Perry, R. P. (2011). Measuring emotions in students' learning and perfomance: the Achievement Emotions Questionnaire (AEQ). Contemporary Educational Psychology, 36 (1), 36-48

Rodríguez-Arteche, I., Bárcena, A.I., Rosa, D. y Martínez-Aznar, M.M. (2019). Aprendizaje indagativo sobre los cambios físicos y químicos en la formación inicial del profesorado de secundaria. Ápice. Revista de Educación Cientifica, 3(2), 1-20. DOI: https://doi. org/10.17979/arec.2019.3.2.4657

Rodríguez Moreno, J., Pro Bueno A. y Molina Jaén, M. D. (2018). Opinión de los docentes sobre el tratamiento de las competencias en los libros de texto de Ciencias de la Naturaleza en Educación Primaria. Revista Eureka sobre Enseñanza y Divulgación de las Ciencias 15 (3), 3102 DOI: https://doi.org/10.25267/Rev_Eureka_ensen_divulg_ cienc.2018.v15.i3.3102

Sánchez, J. A. y Gómez, D. L. (2017) Diseño e implementación de un proyecto de investigación en el aula sobre la fitorremediación de CR (VI) como una estrategia para el desarrollo de competencias científicas investigativas. Biografia, 10 (18) 75-88. https://revistas.pedagogica.edu.co/index.php/bio-grafia/article/view/6887

Schwarz, C. V. y Gwekwerere, Y. N. (2007). Using a Guided Inquiry and Modeling Instructional Framewok (EIMA) to Support Preservice K-8 Science Teaching. Science Education, 91(1), 158-186.

Stewart, J., Cartier, J. L. y Passmore, C. M. (2005). Developing understanding through model-based inquiry. In M. S. Donovan \& J. D. Bransford (Eds.), How students learn (pp. 515 565). Washington D.C.: National Research Council.

Summers, R. y Abd-El-Khalick, F. (2018). Development and validation of an instrument to assess student attitudes toward science across grades 5 through 10. Journal Research Science Teaching 55, 172-2015

Toma, R. B. y Meneses, J. A. (2019) Validation of the single-items Spanish-School Science Attitude Survey (S-SSAS) for elementary education. PLOS ONE, 14 (1) e0209027. DOI: https://doi.org/10.1371/journal.pone.0209027

Toma, R. B, Ortiz-Rivella, J. y Greca, I. (2019). ¿Qué actitudes hacia la ciencia posee el alumnado de Educación Primaria que participa en actividades científicas extracurriculares? Ápice. Revista de Educación Científica, 3 (1), 55-69. DOI: https:// doi.org/10.17979/arec.2019.3.1.4599 
Tamir, P., Nussinovitz, R., y Friedler, Y. (1982). The development and use of a Practical Test Assessment Inventory. Journal of Biological Education, 16, 42-50.

Wang T. L. y Berlin D. (2010). Construction and validation of an instrument to measure taiwanese elementary students' attitudes toward their Science class. International Journal Science Education, 32, 2413-2428.

WINDSCHITL, M. (2003). Inquiry Projects in Science Teacher Education: What Can Investigative Experiences Reveal About Teacher Thinking and Eventual Classroom Practice? Science Education, 87 (1), 112-143.

Zhang D. y Campbell T. (2011) The psychometric evaluation of a three-dimension elementary science attitude survey. Journal of Science Teacher Education. 22, 595-612. 
ANEXO A: Rúbrica para analizar las cinco competencias científicas seleccionadas

\begin{tabular}{|c|c|}
\hline $\begin{array}{l}\text { Competencia } \\
\text { Científica }\end{array}$ & Indicadores o niveles \\
\hline $\begin{array}{l}\text { CCI } \\
\text { Identificar } \\
\text { problemas de } \\
\text { la situación } \\
\text { problemática }\end{array}$ & $\begin{array}{l}\text { N1. No identifica ni plantea problemas susceptibles de contrastación experi- } \\
\text { mental. } \\
\text { N2. Identifica y plantea problemas, pero son poco científicos o inabordables. } \\
\text { N3. Plantea problemas poco importantes o con formulación ambigua o gené- } \\
\text { rica o mal formulado. } \\
\text { N4. Identifica problemas interesantes pero formulados ambiguamente, sin } \\
\text { dejar clara la variable dependiente. } \\
\text { N5. Identifica problemas importantes de investigación o plantea problemas } \\
\text { adecuados y concreta el interrogante dejando clara la variable dependiente. }\end{array}$ \\
\hline $\begin{array}{l}\text { CC II } \\
\text { Formular } \\
\text { hipótesis }\end{array}$ & $\begin{array}{l}\text { N1. No formula o no identifica hipótesis o emite hipótesis sin sentido. } \\
\text { N2. Formula hipótesis sin relación con el problema o los objetivos de la inda- } \\
\text { gación o confunde hipótesis y problemas. } \\
\text { N3. Formula hipótesis ambiguas o con errores de lógica o mal formuladas. } \\
\text { N4. Formula hipótesis que encajan con el o los problemas de indagación } \\
\text { N5. Formula hipótesis que encajan con el problema de investigación y las des- } \\
\text { cribe con referencia a un modelo }\end{array}$ \\
\hline $\begin{array}{l}\text { CC III } \\
\text { Planificar } \\
\text { o realizar } \\
\text { el diseño } \\
\text { experimental }\end{array}$ & $\begin{array}{l}\text { N1. No hay o no propone diseño experimental. Confunde la hipótesis cono el } \\
\text { diseño experimental } \\
\text { N2. El diseño experimental no permite contrastar las hipótesis. No se identifi- } \\
\text { can las variables. Solo propone los materiales. } \\
\text { N3. Los procedimientos de investigación sólo permiten una comprobación } \\
\text { parcial de las hipótesis, sin réplicas ni control. Se confunden las variables. } \\
\text { N4. El diseño experimental ofrece una adecuada comprobación de las hipóte- } \\
\text { sis, pero no propone réplicas ni explicita controles o el control es incompleto } \\
\text { o inadecuado o descripción incompleta del diseño. } \\
\text { N5. El diseño experimental ofrece una adecuada comprobación de las hipó- } \\
\text { tesis, propone réplicas y hay control adecuado. Identificación correcta de las } \\
\text { variables. }\end{array}$ \\
\hline $\begin{array}{l}\text { CC IV } \\
\text { Analizar datos } \\
\text { y elaborar } \\
\text { conclusiones } \\
\text { argumentadas }\end{array}$ & $\begin{array}{l}\text { N1. No se han recogido los datos adecuados } \\
\text { N2. Procesamiento inadecuado o incompleto de los datos. Tablas o gráficos } \\
\text { inadecuados. } \\
\text { N3. Análisis y razonamientos no fundamentados en los datos o basado en da- } \\
\text { tos no fiables. } \\
\text { N4. Análisis adecuado de los datos, aunque incompleto y poco fundamentado } \\
\text { ("simplista"). } \\
\text { N5. Buen procesamiento de los datos y análisis de los mismos bien funda- } \\
\text { mentado. }\end{array}$ \\
\hline $\begin{array}{l}\text { CC V } \\
\text { Comunicación y } \\
\text { argumentación } \\
\text { de la indagación }\end{array}$ & $\begin{array}{l}\text { N1. No comunica correctamente el proceso indagatorio y no argumenta debi- } \\
\text { damente. } \\
\text { N2. Tiene algunas dificultades en relatar el proceso indagatorio y en argu- } \\
\text { mentar las conclusiones en base a las evidencias obtenidas. } \\
\text { N3. En general, comunica correctamente lo logrado en las fases de la inda- } \\
\text { gación, pero tiene dificultades en argumentar las conclusiones en base a las } \\
\text { evidencias obtenidas. } \\
\text { N4. Comunica con fluidez y argumenta algunas conclusiones, pero se detecta } \\
\text { alguna dificultad en la comprensión del conocimiento científico tratado y en } \\
\text { el proceso indagatorio. } \\
\text { N5. Comunica con fluidez demostrando comprender el proceso de indagación } \\
\text { seguido y argumenta las conclusiones en base a las evidencias obtenidas. }\end{array}$ \\
\hline
\end{tabular}


ANEXO B: Cuestionario para evaluar actitudes y emociones hacia la Ciencia (SSAS y AEQ)

1. Quiero apuntarme a clases de Ciencias en secundaria (SD Totalmente en desacuerdo - totalmente en acuerdo).

2. Me gusta ir a clases de Ciencias (SD Totalmente en desacuerdo - totalmente en acuerdo).

3. Me da miedo equivocarme en clases de Ciencias, así que mejor no digo nada. (SD Totalmente en desacuerdo - totalmente en acuerdo).

4. Pienso que las clases de Ciencias son (SD Aburridas - Divertidas).

5. Quiero aprender sobre la electricidad y saber cómo se usa en una casa (SD Totalmente en desacuerdo - totalmente en acuerdo).

6. Disfruto cuando estoy en clases de Ciencias (SD Totalmente en desacuerdo - totalmente en acuerdo).

7. Me cuesta terminar las tareas para la clase de Ciencias (SD Totalmente en desacuerdo - totalmente en acuerdo).

8. Al terminar la clase de Ciencias, ya estoy deseando que llegue la próxima (SD Totalmente en desacuerdo - totalmente en acuerdo).

9. Pienso que soy muy bueno en las clases de Ciencias (SD Totalmente en desacuerdo - totalmente en acuerdo).

10. En clase de Ciencias me siento muy nervioso (SD Totalmente en desacuerdo - totalmente en acuerdo).

11. Un trabajo como científico sería interesante (SD Totalmente en desacuerdo - totalmente en acuerdo).

12. Estoy motivado a ir a clase de Ciencias porque es interesante (SD Totalmente en desacuerdo - totalmente en acuerdo).

13. Me siento mal cuando no entiendo algo en la clase de Ciencias (SD Totalmente en desacuerdo - totalmente en acuerdo).

14. Para mis futuros estudios, el conocimiento de las clases de Ciencias es: (SD Inútil - Útil).

15. Disfruto tanto en clases de Ciencias que podría estar horas aprendiendo Ciencias (SD Totalmente en desacuerdo - totalmente en acuerdo).

16. Me preocupan las clases de Ciencias (SD Totalmente en desacuerdo - totalmente en acuerdo).

17. La Ciencia ayuda a mejorar la vida (SD Totalmente en desacuerdo - totalmente en acuerdo).

18. Tengo miedo de las clases de Ciencias (SD Totalmente en desacuerdo - totalmente en acuerdo).

19. Para mi vida diaria, creo que las clases de Ciencias son: (SD Poco importantes - muy importantes).

20. Quiero aprender sobre las plantas de mi entorno. 
ANEXO C: Evaluación de las competencias asociadas a las prácticas científicas

Los hermanos Pablo y Jaime tienen ocho y seis años respectivamente. Han decidido hacer un tirachinas cada uno para jugar a derribar latas de refrescos vacías colocadas encima de una mesa, situada a una distancia de 10 metros de ellos. Cada uno ha utilizado distintos materiales para construirlo.

Para derribar las latas, Pablo y Jaime utilizan garbanzos como proyectil, colocándolos en el extremo del tirachinas. Los dos tiraron varias veces a las latas y siempre el proyectil de Jaime llegaba a más distancia de donde estaban las latas, derribándolas si tenía buena puntería o sobrepasándolas si su puntería era mala, mientras que el de Pablo no llegaba nunca a las latas.
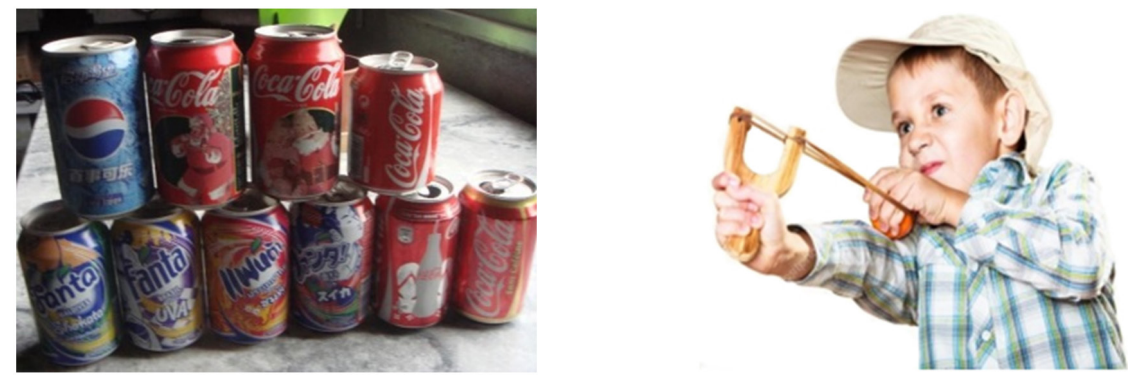

CUESTIÓN 1 Plantea tres preguntas de investigación relacionadas con la situación anterior.

CUESTIÓN 2 ¿Qué experimento harías para comprobar que un garbanzo colocado en el extremo del tirachinas del niño de la figura anterior llegará más o menos lejos dependiendo de lo que alargues la goma?

CUESTIÓN 3 ¿Qué experimento harías para comprobar que un garbanzo colocado en el extremo del tirachinas del niño de la figura anterior llegará más o menos lejos dependiendo del tipo de goma que se utilice?

CUESTIÓN 4 Sabes cómo construir un circuito para que brille una bombilla. Ahora te pedimos que formules dos hipótesis que respondan a la siguiente pregunta:

¿Cómo se puede conseguir que el brillo de una bombilla cambie?

CUESTIÓN 5 En la tabla se presentan los valores de la temperatura del agua colocada en un recipiente, puesto al Sol durante 15 minutos y el estado de agregación en el que se encuentra.

\begin{tabular}{|c|c|c|}
\hline $\begin{array}{c}\text { Tiempo transcurrido } \\
\text { (minutos) }\end{array}$ & Estado de agregación & $\begin{array}{c}\text { Temperatura } \\
\text { del agua }\left({ }^{\circ} \mathbf{C}\right)\end{array}$ \\
\hline 0 & sólido & -3 \\
\hline 2 & sólido & -2 \\
\hline 4 & sólido & -1 \\
\hline 6 & sólido y líquido & 0 \\
\hline 8 & sólido y líquido & 0 \\
\hline 10 & sólido y líquido & 0 \\
\hline 12 & líquido & 1 \\
\hline 15 & líquido & 2 \\
\hline
\end{tabular}


